\title{
Geometrical design of variable ratio tooth profile based on Boolean subtraction operation and a novel modification method
}

\author{
Ziru Niu ${ }^{1,2}$, Jianlei Li ${ }^{1}$, Shuai Xin ${ }^{1}$, Liangliang Zou ${ }^{1,2}$, Yuhua $\mathrm{Li}^{1,2}$, Jialin $\mathrm{Hou}^{1,2}$, \\ Ming $\mathrm{Tu}^{3,4^{*}}$, Ting Sun ${ }^{5}$ \\ (1. College of Mechanical and Electronic Engineering, Shandong Agricultural University, Tai'an 271018, Shandong, China; \\ 2. Shandong Provincial Key Laboratory of Horticultural Machinery and Equipment, Tai'an 271018, Shandong, China; \\ 3. College of Engineering, Huazhong Agricultural University, Wuhan 430070, China; \\ 4. Key Laboratory of Agricultural Equipment in Mid-lower Reaches of the Yangtze River, Ministry of Agriculture and Rural Affairs, \\ Wuhan 430070, China; 5. Shandong Huasheng Pesticide Machinery Co., Ltd, Linyi 276017, Shandong, China)
}

\begin{abstract}
Variable transmission ratio racks show great potential in rice transplanters as a key component of variable transmission ratio steering to balance steering portability and sensitivity. The objective of this study was to develop a novel geometrical design method to achieve quick, high-quality modeling of the free curvilinear tooth profile of a variable transmission ratio rack. First, a discrete envelope motion 3D model was established between the pinion-sector and the variable transmission ratio rack blank based on the mapping relationship between the rotation angle of the pinion-sector and the displacement of the rack, according to the variable transmission ratio function. Based on the loop Boolean subtraction operation, which removed the pinion-sector from the rack blank during all moments of the discrete motion process, the final complex changing tooth shape of the variable transmission ratio rack was enveloped. Then, since Boolean cutting residues made the variable ratio tooth surface fluctuant and eventually affected the precision of the model, this study proposed a modification method for establishing a smooth and continuous tooth profile. First, a novel fitting algorithm used approximate variable ratio tooth profile points extracted from the Boolean cutting marks and generated a series of variable ratio tooth profiles by utilizing B-spline with different orders. Next, based on a transmission stability simulation, the variable ratio tooth profile with optimal dynamic performance was selected as the final design. Finally, tests contrasting the transmission stability of the machining samples of the initial variable ratio tooth profile and the final variable ratio tooth profile were conducted. The results indicated that the final variable ratio tooth profile is more effective than the initial variable ratio tooth profile. Therefore, the proposed variable ratio tooth profile modeling and modification method for eliminating Boolean cutting residues and improving surface accuracy is proved to be feasible.
\end{abstract}

Keywords: rice transplanter, steering, variable ratio tooth profile, variable ratio curve, Boolean subtraction operation, transmission stability

DOI: $10.25165 /$ j.ijabe.20201305.4884

Citation: Niu Z R, Li J L, Xin S, Zou L L, Li Y H, Hou J L, et al. Geometrical design of variable ratio tooth profile based on Boolean subtraction operation and a novel modification method. Int J Agric \& Biol Eng, 2020; 13(5): 125-133.

\section{Introduction}

A steering system with a constant transmission ratio is unable to provide the comfortable steering ratio that rice transplanters need

Received date: 2018-12-25 Accepted date: 2020-07-12

Biographies: Ziru Niu, PhD, Associate Professor, research interest: digital design and manufacturing; intelligent agricultural machinery equipment, Email: cherokeesaab@163.com; Jianlei Li, Master candidate, research interest: intelligent agricultural machinery equipment, Email: jianlei0927@163.com; Shuai Xin, Master candidate, research interest: digital design and manufacturing, Email: 15753833500@163.com; Liangliang Zou, Doctoral student, Lecturer, research interest: intelligent agricultural machinery equipment, Email: zouliangliang1986@163.com; Yuhua Li, Doctoral student, Lecturer, research interest: intelligent agricultural machinery equipment, Email: 18218148@qq.com; Jialin Hou, PhD, Professor, research interest: intelligent agricultural machinery equipment, Email: jlhou@sdau.edu.cn; Ting Sun, Bachelor, Engineer, research interest: intelligent agricultural machinery equipment, Email: 13573966929@163.com.

*Corresponding author: Ming Tu, $\mathrm{PhD}$, Assistant Professor, research interest: intelligent agricultural machinery equipment, No.1, Shizishan Street, Hongshan District, Wuhan 430070, China. Tel: +86-13971626490, Email: mingtu@mail.hzau.edu.cn. in a paddy field with high moisture and low carrying capacity. A variable ratio steering system should be applied in transplanters to ensure motion stability when driving in a straight line ${ }^{[1]}$. A variable ratio steering system makes transplanters respond quickly when starting a turn, and also reduces effort near the wheel turning limits $^{[2]}$.

Variable ratio steering systems are divided into three categories at present, including the steer-by-wire system (SBW), the electronic controlled steering system (ECS) and the mechanical variable ratio steering system (MVRS). The SBW is operated by the control unit, which can directly drive a steering motor to implement the change in the wheel turning angle; however, due to the lack of a mechanical connection, its safety needs to be improved $^{[3]}$. The ECS has successfully integrated a mechanical steering system and a set of double planetary gear mechanisms driven by an electric motor to provide an additional wheel angle to regulate transmission ratio, so it is more practicable $\mathrm{e}^{[4]}$, but the extra gear mechanism increases manufacturing difficulty and energy consumption. Both the SBW and the ESC use the steering motor to provide limited steering force, but the force is not sufficient to meet the steering requirements of rice transplanters under severe 
working conditions. The MVRS uses gears with variable ratio to achieve transmission ratio variation while retaining the original mechanical structure used by rice transplanters ${ }^{[5,6]}$. Therefore, the MVRS is appropriate for use in rice transplanters to deal with complicated and variable applied environments because of its low cost, high reliability and durability.

Gears with variable ratio have been a general concern and a challenge from both a theoretical and a technological point of view. The most innovative solution is to break the linear profile of the rack. For a continuous variable ratio, the rack requires a free curvilinear tooth profile, which is called the variable ratio tooth profile (VRTP) ${ }^{[7-10]}$. More specifically, it is an envelope surface generated by the involute tooth profile and moving according to a variable transmission ratio curve, so the involute gear and the enveloped rack constitute a novel gear pair with VRTP. Unlike non-circular gears, which have a varied pitch radius but a uniform tooth thickness, the tooth thickness of the variable transmission ratio rack varies along the length to achieve variable ratio drive. Thus, the variable transmission ratio rack has a more complex tooth shape than non-circular gears ${ }^{[10]}$.

The novel gear pair with VRTP still follows conjugate meshing characteristics, so the design ${ }^{[11]}$, modeling ${ }^{[12,13]}$, analysis $^{[14,15]}$ and manufacturing ${ }^{[16,17]}$ methods for traditional gears are also applicable. Meshing theory has played a momentous role in gear geometry. Litvin et al. ${ }^{[18-23]}$ investigated the gear design, geometry analysis, manufacturing and processing of gear drive in depth and made significant progress in promoting the development of non-circular gears. Liu et al. ${ }^{[2]}$ presented an undulating face gear that can transmit varying angular velocities, and derived the equation for the tooth profile through geometry analysis. However, the research results found in the literature ${ }^{[7,25,26]}$ indicated that a variety of shortcomings would result from using meshing theory to design VRTP: (1) when the coincidence degree is greater than 1 , the solution of the meshing equation will not be unique; (2) the exact values of the boundary points of the addendum and dedendum cannot be obtained due to the complexity of VRTP boundary conditions; (3) the generated isoparametric meshing lines from the tooth surface representation are non-equidistant and the generated tooth profile points on the lines are unevenly distributed across the surface of the tooth, meaning that these points cannot be used to accurately obtain a solid model of VRTP.

The CAD method, which obtains a solid model by simulating the process of gear manufacturing, has been developed as one type of advanced technology. Mazak et al. ${ }^{[27]}$ extended the manufacturing simulation based on planar penetration to generate the processes of bevel gears for the first time. Using the principle of power skiving for the internal gear, Guo et al. ${ }^{[28]}$ obtained an internal spur gear. A Boolean subtraction operation method based on the envelope principle was proposed by Lin et al. ${ }^{[29]}$ to obtain an accurate tooth profile for internal non-circular gears. However, it is still a challenge to apply the CAD method to VRTP. The problem comprises two main aspects: (1) the generated VRTP is not a continuous surface, and cannot be used directly for further studies, such as finite element analysis; and (2) accurate tooth profile points cannot be extracted from the generated VRTP, so the theoretical tooth profile cannot be obtained by fitting these points. Therefore, the primary problem for the next body of research is to establish an accurate solid model of VRTP.

This study proposed an advanced CAD/CAE integration method to design VRTP, which was continuous and smooth. The feasibility of the method was subsequently verified through a transmission stability test using the processed samples.

\section{Generation of initial rack with VRTP}

As illustrated in Figure 1, the variable ratio steering installed on rice transplanters consists of a screw-nut pair and a variable ratio rack and pinion-sector pair. The transmission ratio of the screw-nut pair is constant, while the variable transmission ratio is obtained by means of a variable ratio rack and pinion-sector pair composed of an involute pinion-sector and a conjugated rack with VRTP. VRTP is designed as the envelope surface of the involute pinion-sector tooth surface, per the constraints of a variable transmission ratio curve. There are three generation processes to determine VRTP: (1) the involute pinion-sector is designed by CAD software; (2) the discrete motion relationship between the variable ratio rack and the pinion-sector is digitally expressed according to a variable transmission ratio function; and (3) VRTP is generated with the Boolean subtraction operation modeling method.

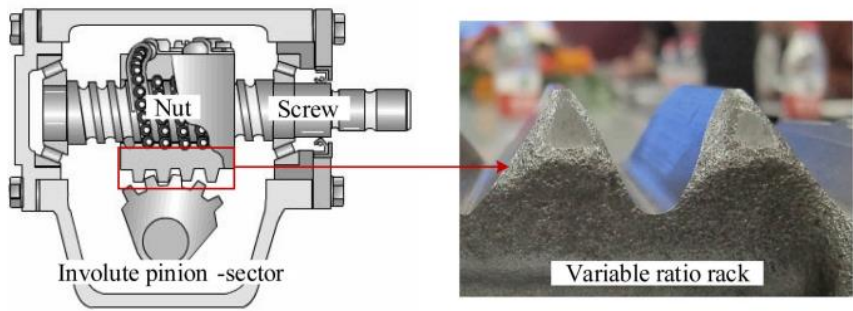

Figure 1 Steering and variable ratio tooth profile

\subsection{Modeling of involute beveloid pinion-sector}

Compared to straight gears, beveloid gears have the advantages of large coincidence degree and stable transmission. The involute beveloid pinion-sector reserving three teeth is designed as shown in Figure 2. The cone angle $\delta$ of each tooth from the large end to the small end is $5^{\circ}$, as shown in Figure $2 \mathrm{c}$, and the left tooth and the right tooth are symmetrical around the symmetry plane $L$ of the middle tooth, as shown in Figures $2 \mathrm{a}$ and $2 \mathrm{~b}$. The new design is created by artificially changing the positions of the involute starting points. On the large end, the involute starting points of the left profile of the middle tooth, the right profile of the left tooth, and the left profile of the left tooth are denoted by $A, B$ and $C$, respectively, as shown in Figure 2a. Those distances to the plane $L$ are $A A^{\prime}=8.99 \mathrm{~mm}, B B^{\prime}=15.74 \mathrm{~mm}$ and $C C^{\prime}=27.42 \mathrm{~mm}$, separately Similarly, on the small end, the corresponding involute starting points are represented by $a, b$ and $c$, and the corresponding distances are $a a^{\prime}=6.67 \mathrm{~mm}, b b^{\prime}=17.68 \mathrm{~mm}$ and $c c^{\prime}=26.32 \mathrm{~mm}$, respectively, as shown in Figure $2 b$. Other parameters of the involute beveloid pinion-sector include the base circle $r_{\mathrm{b}}$, the addendum circle $r_{\mathrm{a}}$ and the dedendum circle $r_{\mathrm{f}}$, and the corresponding values are $r_{\mathrm{b}}=30.16 \mathrm{~mm}, r_{\mathrm{a}}=46.02 \mathrm{~mm}$ and $r_{\mathrm{f}}=$ $27.31 \mathrm{~mm}$.

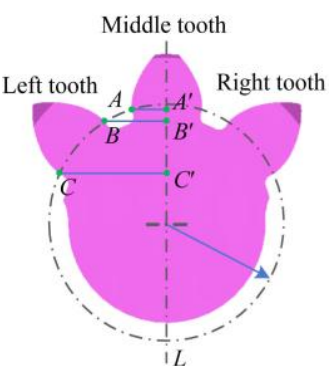

a. Large end

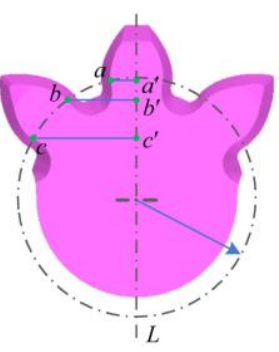

b. Small end

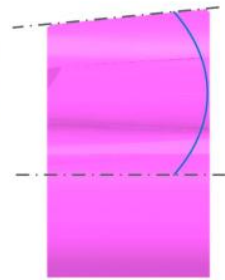

c. Cone angle
Figure 2 Involute beveloid pinion-sector 


\subsection{Digital expression of variable transmission ratio curve}

The CAD modeling method based on the Boolean subtraction operation begins by digitally reproducing the discrete motion relationship between the variable ratio rack and the pinion-sector pair to simulate the enveloping movement. The relationship between the displacement of the rack and the rotation angle of the involute beveloid pinion-sector can be reflected through the variable transmission ratio curve of the gears.

The transmission ratio of a variable ratio rack and a pinion-sector pair is defined by the ratio of the line speed of the rack and the angular speed of the pinion-sector and can be written as Equation (1).

$$
i=\frac{d s}{d \varphi}
$$

where, $s$ is the displacement of the rack, mm; and $\varphi$ is the rotation angle of the pinion-sector, $\left({ }^{\circ}\right)$.

At present, a universal variable transmission ratio function can be expressed as Equation (2), and the related curve is shown in Figure 3 .

$$
i(\varphi)=\left\{\begin{array}{cc}
i_{1} & \left(|\varphi|<\varphi_{1}\right) \\
\frac{i_{1}-i_{2}}{2} \cos \left(\frac{\pi|\varphi|}{\varphi_{2}-\varphi_{1}}-\frac{\pi \varphi_{1}}{\varphi_{2}-\varphi_{1}}\right)+\frac{i_{1}+i_{2}}{2} & \left(\varphi_{1} \leq|\varphi| \leq \varphi_{2}\right) \\
i_{2} & \left(\varphi_{2}<\varphi \mid \leq \varphi_{3}\right)
\end{array}\right.
$$

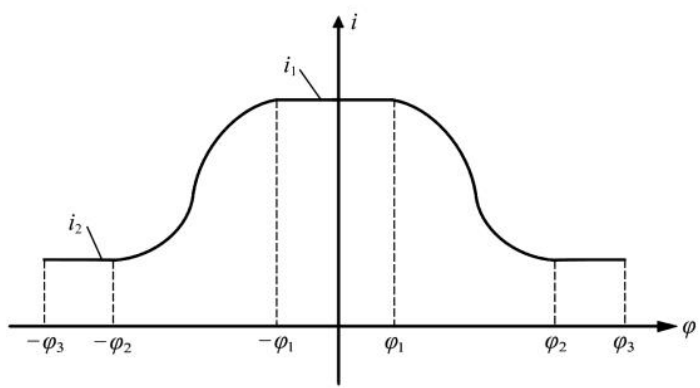

Figure 3 Variable ratio curve

Equation (2) and Figure 3 show that the variable transmission ratio curve is segmented and symmetrical. When the rotation angle $\varphi \in\left(-\varphi_{1}, \varphi_{1}\right)$, the transmission ratio is a constant $i_{1}$; when the rotation angle $\varphi \in\left(-\varphi_{3}, \varphi_{2}\right] \mathrm{U}\left[\varphi_{2}, \varphi_{3}\right)$, the transmission ratio is a constant $i_{2}$. The curves between $i_{1}$ and $i_{2}$ are the core for a variable transmission ratio curve when the rotation angle $\varphi \in\left[-\varphi_{2}\right.$, $\left.\varphi_{1}\right] \mathrm{U}\left[\varphi_{1}, \varphi_{2}\right]$.

While the rotation angle of the pinion-sector changes from $\varphi$ to $\varphi+\Delta \varphi$ within the specified range, the rack is displaced a certain $\Delta s$, which can be obtained by calculating the definite integral of the variable transmission ratio function in rotation angle interval $[\varphi, \varphi+\Delta \varphi]$. Combined with Equations (1) and (2), the discretized displacements of the rack can be digitally calculated as

$$
\begin{aligned}
& \Delta s(k)= \\
& \left\{\begin{array}{lr}
i_{1} \Delta \varphi & \left(1<|i|<\frac{\varphi_{1}}{\Delta \varphi}\right) \\
\frac{i_{1}-i_{2}}{2 \omega}(\sin (\omega \Delta \varphi|i|-\omega \varphi)-\sin (\omega \Delta \varphi|i-1|-\omega \varphi))+\frac{i_{1}+i_{2}}{2} \Delta \varphi & \left(\frac{\varphi_{1}}{\Delta \varphi} \leq|i| \leq \frac{\varphi_{2}}{\Delta \varphi}\right) \\
i_{2} \Delta \varphi & \left(\frac{\varphi_{2}}{\Delta \varphi}<|i| \leq \frac{\varphi_{3}}{\Delta \varphi}\right)
\end{array}\right.
\end{aligned}
$$

where, $\omega=180^{\circ} /\left(\varphi_{2}-\varphi_{1}\right)$. The other symbols are explained in Equations (1) and (2).

In this paper, a variable transmission ratio function is given as Equation (4), and the digital displacement of the rack is expressed as Equation (5).

$$
\begin{gathered}
i=\frac{1}{24}\left(-1.5 \cos \left(\frac{\pi \varphi}{60}\right)+16.75\right) \quad\left(|\varphi| \leq 47^{\circ}\right) \\
\Delta s=\frac{1}{24}\left(-\frac{90}{\pi}\left(\sin \left(\frac{\pi}{60}(\varphi+\Delta \varphi)\right)-\sin \left(\frac{\pi \varphi}{60}\right)\right)+16.75 \Delta \varphi\right)
\end{gathered}
$$

\subsection{Direct generation of initial rack with VRTP}

As illustrated in Figure 4a, using the involute beveloid pinion-sector obtained in Section 2.1, the virtual assembly model between a rack blank and the pinion-sector can be structured in CAD software. The assembly angle $\delta$ is $5^{\circ}$.

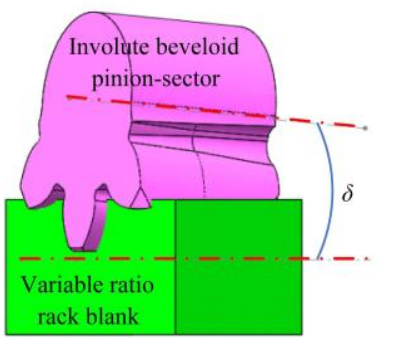

a. Assembly model

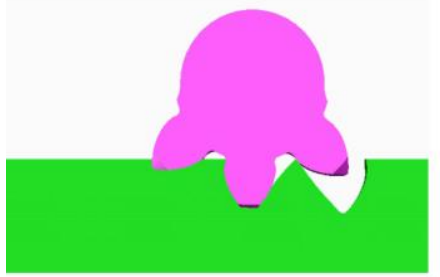

c. $\varphi=0 \mathrm{deg}$

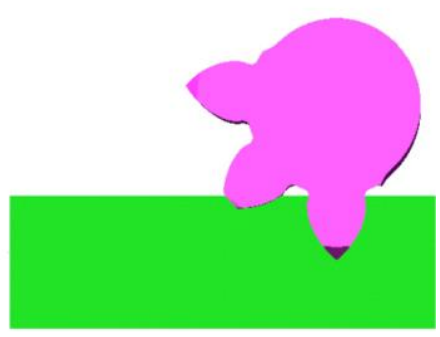

b. $\varphi=-47 \mathrm{deg}$

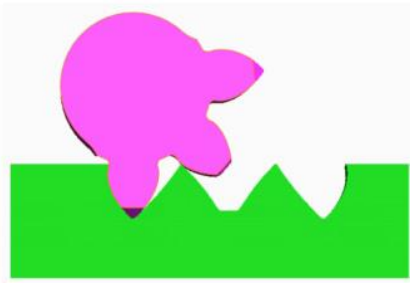

d. $\varphi=47 \mathrm{deg}$

Figure 4 Boolean subtraction modeling process for variable ratio tooth profile

The macro-programming that can achieve the Boolean subtraction operation is adopted to establish a solid model of VRTP First, the involute beveloid pinion-sector rotates around its own axis with a rotation angle increment $\Delta \varphi$; the rack blank correspondingly creates a displacement $\Delta s$. Then the single Boolean subtraction operation is performed to remove the pinion-sector from the rack blank. According to the digitized motion relationship between the variable ratio rack and the pinion-sector, the loop operation of the two steps above is implemented by removing the pinion-sector from the rack blank during all moments of the discrete motion process. In addition, the three representative moments in the mashing motion of the variable ratio rack and the pinion-sector pair are shown in Figure 4, which are $\varphi=-47^{\circ}, \varphi=0^{\circ}$ and $\varphi=47^{\circ}$. The initial rack is shown in Figure 5.

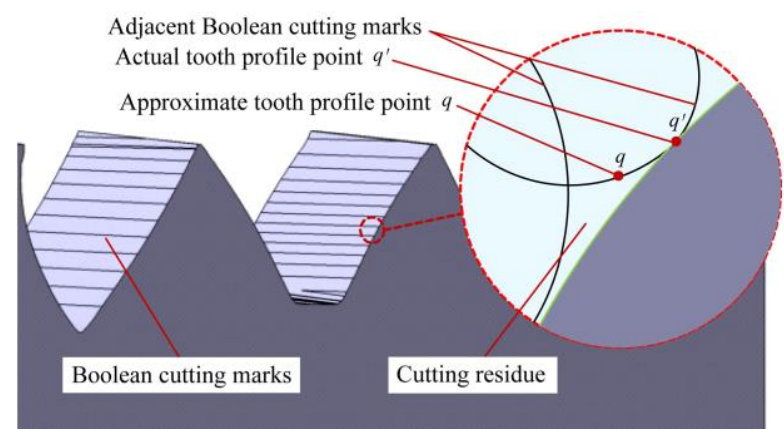

Figure 5 Initial rack model with variable ratio tooth profile 
Experiments have shown that the generated model of VRTP is satisfactory when the rotation angle increment of the pinion-sector is $\Delta \varphi=1^{\circ}-2^{\circ}$. According to the result shown in Figure 5, since the continuous motion relationship can only be realized discretely by CAD software, the VRTP of the initial rack is a discontinuous surface comprising many Boolean cutting marks. The cutting residue left on the VRTP between two adjacent cutting marks makes the tooth surface fluctuant and eventually affects the precision of the model.

With the obtained VRTP, neither CAE nor CNC machining can be implemented flawlessly. Thus, an advanced modification method is necessary to regenerate the tooth surface of the rack to eliminate cutting marks, obtain a continuous tooth profile and improve model accuracy. The proposed approach used the following three steps.

(1) The approximate VRTP points of the initial rack are extracted from the Boolean cutting marks. Since the actual VRTP point $q^{\prime}$ is difficult to lock into position, the midpoint of each Boolean cutting mark on the end of the initial rack is selected as the approximate VRTP point $q$.

(2) The fitting algorithm is studied to fit the approximate VRTP points based on the B-spline curves with different orders; then a series of modified racks with continuous VRTP is obtained.

(3) Transmission stability simulations are carried out with these modified racks, and the rack with the best transmission stability is selected as the final VRTP design.

\section{Modification method based on B-spline curve}

As mentioned in Section 2.3, the flow chart of the proposed modification method is shown in Figure 6 and is described in detail over three subsections.

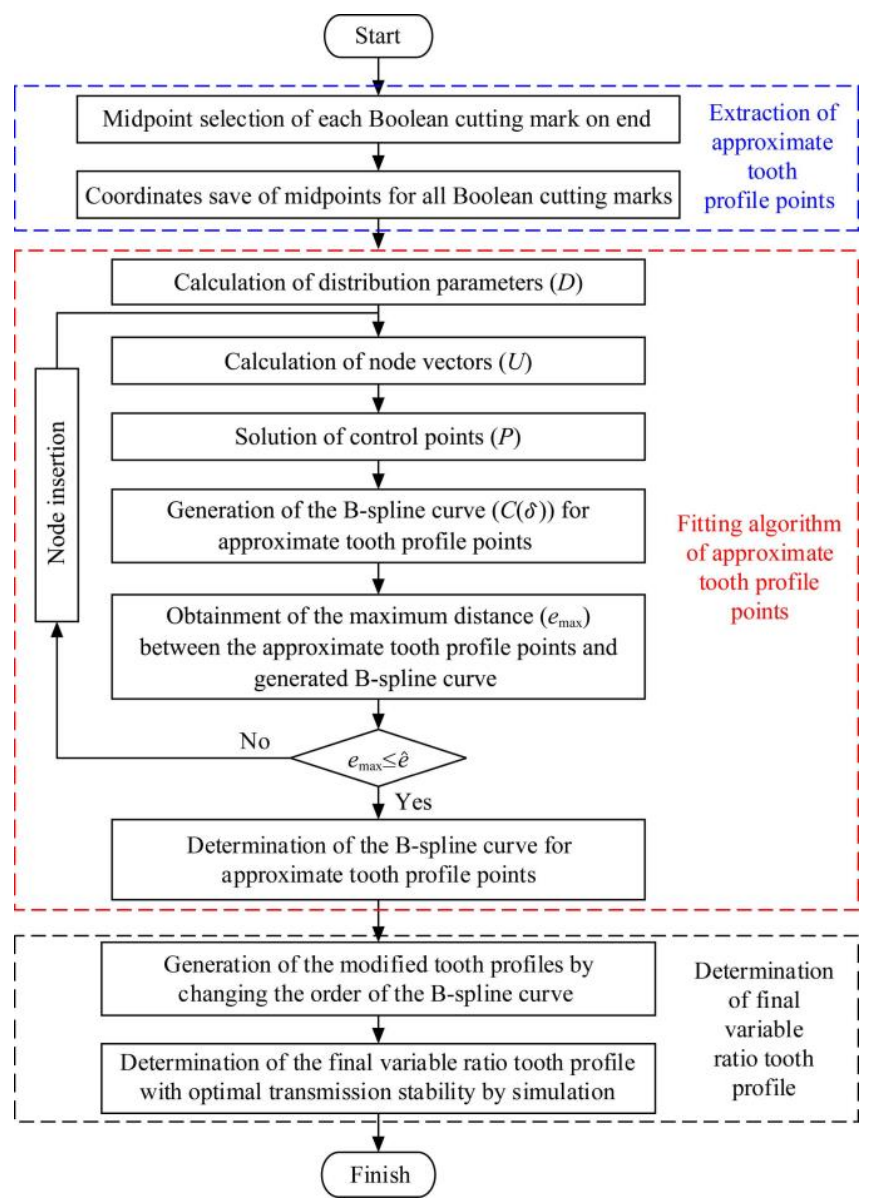

Figure 6 Flow chart of modification method

\subsection{Extraction of approximate tooth profile points}

It is hard to determine the exact locations of the tooth profile points of VRTP through the cutting marks, though these points are distributed along with the Boolean cutting marks of the initial rack. In this case, extracting the approximate tooth profile points is reasonable and feasible. The extraction process mainly consists of two steps: (1) Select the midpoint of each Boolean cutting mark on the end of the initial rack; (2) Save the coordinate of the midpoint as the approximate location of the corresponding tooth profile point. After all Boolean cutting marks have been traversed, the approximate tooth profile points can be obtained. The approximate tooth profile points can be recorded mathematically with $Q$

$$
Q=\left\{q_{i}\right\}, i \in[0, m]
$$

where, $q_{i}$ denotes the element coordinate, and the total number of elements is $m+1$.

\subsection{Fitting algorithm of approximate tooth profile points}

The design theory of the B-spline curve is adopted to approach the approximate tooth profile points within a specified fitting accuracy because of the high advantage B-spline offers in representing free curves. The B-spline curve can be formulated by

$$
C(\delta)=\sum_{i=0}^{n} N_{i, \lambda}(\delta) \cdot p_{i}, \quad \delta \in[0,1]
$$

where, $\delta$ varying from 0 to 1 denotes the independent variable; $\lambda$ $(\lambda=3,4,5, \cdots)$ is the function order, $p_{i}$ denotes an element of the control points $P$, and $N_{i, \lambda}(\delta)$ is the basic function of the B-spline. The total numbers of control points and basis functions are both $n+1$. The expression of $N_{i, \lambda}(\delta)$ is

$$
N_{i, \lambda}(\delta)=\frac{\delta-u_{i}}{u_{i+\lambda}-u_{i}} N_{i, \lambda-1}(\delta)+\frac{u_{i+\lambda+1}-\delta}{u_{i+\lambda+1}-u_{i+1}} N_{i+1, \lambda-1}(\delta), i \in[0, n]
$$

where, $u_{j}$ denotes the element of the node vectors $U$. It is necessary to emphasize that the total number is always $n+\lambda+2$, that is, the subscript $j$ varies from 0 to $n+\lambda+1$.

The calculations of the node vectors $U$ and the control points $P$ can uniquely determine the formula of the $\lambda$-th B-spline curve. On the other hand, increasing the value of $n$ that is, adding the quantities of the control points $P$ and the node vectors $U$ could improve the fitting accuracy.

Based on the above analyses, a fitting algorithm, as shown in Figure 6, is proposed to achieve a quick fitting of the approximate tooth profile points extracted in this paper. In this algorithm, the close loop consisting of the node insertion feedback and the calculation of the node vectors $U$ and the control points $P$ aims to maintain a high preset fitting accuracy. In this close loop, the least square method is adopted to solve the control points $P$, and the maximum value of the distances between the approximate tooth profile points $Q$ and the generated curve $C(\delta)$ is required to be lower than the preset accuracy $\hat{e}$, which is $0.03 \mathrm{~mm}$.

In addition, mathematical processing is very significant when performing this algorithm. Based on the solution methods of the B-spline curve, core calculation formulas are shown in the following four points.

(1) Calculation of distribution parameters

The distribution parameters $D$ are utilized to describe the density of the distribution of approximate tooth profile points. This is necessary for the calculations of the node vectors $U$ and the control points $P$, and can be completely solved by $Q$. The calculation of distribution parameters $D$ is

$$
D=\left\{d_{i}\right\}, i \in[0, m]
$$




$$
d_{i}=\left\{\begin{array}{lc}
0 & i=0 \\
d_{i-1}+\frac{\left|q_{i}-q_{i-1}\right|}{\sum_{j=1}^{m}\left|q_{j}-q_{j-1}\right|} & i \in[1, m]
\end{array}\right.
$$

(2) Calculation of node vectors

The node vectors $U$ can reflect the scattered condition of the distribution parameters $D$, and every interval of the node vectors $U$ should contain at least one element of the distribution parameters $D$. The corresponding computational expression is

$$
U=\left\{u_{i}\right\}, \quad i \in[0, n+\lambda+1]
$$

where,

$$
u_{i}=\left\{\begin{array}{lc}
0 & i \in[0, \lambda] \\
(1+\operatorname{int}(\zeta)-\zeta) \cdot d_{\mathrm{int}(\zeta)-1}+(-\operatorname{int}(\zeta)) \cdot d_{\mathrm{int}(\zeta)} & i \in[\lambda+1, n] \\
1 & i \in[n+1, n+\lambda+1]
\end{array}\right.
$$

in which $\zeta=\frac{(i-\lambda) \cdot(m+1)}{n-\lambda+1}$, and $\operatorname{int}(\zeta)$ denotes the integer portion of $\zeta$.

(3) Calculation of control points

The least squares method is utilized to solve the control points $P$, and the formula can be expressed as

$$
\min \left(\sum_{i=0}^{m}\left|q_{i}-C\left(d_{i}\right)\right|^{2}\right)
$$

Equation (13) can be transformed into $\partial f / \partial D=0$ under the conditions of the function setting with $f=\sum_{i=1}^{m-1}\left|q_{i}-C\left(d_{i}\right)\right|^{2}$ and the existing solutions with $q_{0}=C\left(d_{0}\right)$ and $q_{m}=C\left(d_{m}\right)$. The remaining control points $P^{*}, P^{*}=\left[p_{1}, p_{2}, p_{3}, \ldots, p_{n-1}\right]$, in vector form, can be solved by

$$
P^{*}=\left[N^{T} N\right]^{-1} \cdot R
$$

where, the matrix $\left(N^{T} N\right)$ is always positive definite [30], and

$$
\begin{aligned}
N=\left[\begin{array}{cccc}
N_{1, \lambda}\left(d_{1}\right) & N_{2, \lambda}\left(d_{1}\right) & \ldots & N_{n-1, \lambda}\left(d_{1}\right) \\
N_{1, \lambda}\left(d_{2}\right) & N_{2, \lambda}\left(d_{2}\right) & \ldots & N_{n-1, \lambda}\left(d_{2}\right) \\
\vdots & \vdots & & \vdots \\
N_{1, \lambda}\left(d_{m-1}\right) & N_{2, \lambda}\left(d_{m-1}\right) & \ldots & N_{n-1, \lambda}\left(d_{m-1}\right)
\end{array}\right] \\
R=\left[\begin{array}{l}
\sum_{i=1}^{m-1} \Gamma_{i} N_{1, \lambda}\left(d_{i}\right) \\
\sum_{i=1}^{m-1} \Gamma_{i} N_{2, \lambda}\left(d_{i}\right) \\
\vdots \\
\sum_{i=1}^{m-1} \Gamma_{i} N_{n-1, \lambda}\left(d_{i}\right)
\end{array}\right]
\end{aligned}
$$

where, $\Gamma_{i}=q_{i}-N_{0, \lambda}\left(d_{0}\right) \cdot p_{0}-N_{i, \lambda}\left(d_{m}\right) \cdot p_{n}, i \in[1, m-1]$.

(4) Judgment of fitting accuracy

The maximum value $e_{\max }$ of the distances between the approximate tooth profile points $Q$ and the generated B-spline curve $C(\delta)$ should be lower than the preset accuracy $\hat{e}$. The corresponding formula is

$$
e_{\max } \leq \hat{e}
$$

where,

$$
e_{\max }=\max \left(\left\{\min \left(\left|q_{i}-C(\delta)\right|\right), \delta \in[0,1]\right\}, i \in[0, m]\right)
$$

\subsection{Determination of final rack}

\subsubsection{Application of fitting algorithm}

After extracting the approximate tooth profile points from
Boolean cutting marks, the B-spline tooth profile curve that meets the preset accuracy $\hat{e}=0.03 \mathrm{~mm}$ can be obtained according to the fitting algorithm detailed in Section 3.2. Then the continuous VRTP is generated by stretching the fitting curve along the width of the tooth in CAD software. Starting from $\lambda=3$, the increment of $\lambda$ is $1(\lambda=3,4,5, \cdots)$, and the corresponding fitting curves and several modified racks are generated. Take the fifth-order B-spline fitting curve shown in Figure 7a, for example, and the corresponding modified rack shown in Figure $7 \mathrm{~b}$.

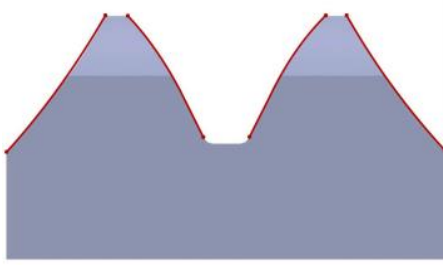

a. Fifth order B-spline fitting curve

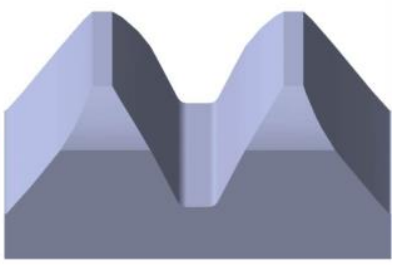

b. Modified rack
Figure 7 Variable ratio tooth profile of the fifth order B-spline fitting curve

\subsubsection{Simulation and analysis of transmission stability}

Transmission stability, which can be estimated by transmission ratio and transmission acceleration, is considered a significant basis for verifying the validity of those variable transmission ratio rack models generated by the fitting curves of different orders. Therefore, transmission stability simulations are made by CAE software with the objective of finding the rack with the best transmission stability. Based on the assembly relationship described in Section 2.3, the assembly models can be constructed. With the assembly models, the simulation models are obtained under the following conditions: the material is steel with the properties of Young's modulus $E=2.07 \times 10^{5} \mathrm{~N} / \mathrm{mm}^{2}$; Poisson's ratio is $v=0.29$; a rack input speed is $2.5 \mathrm{~mm} / \mathrm{s}$; a torque of $200 \mathrm{Nm}$ has been applied to the pinion-sector. As an example, the simulation model is shown in Figure 8.

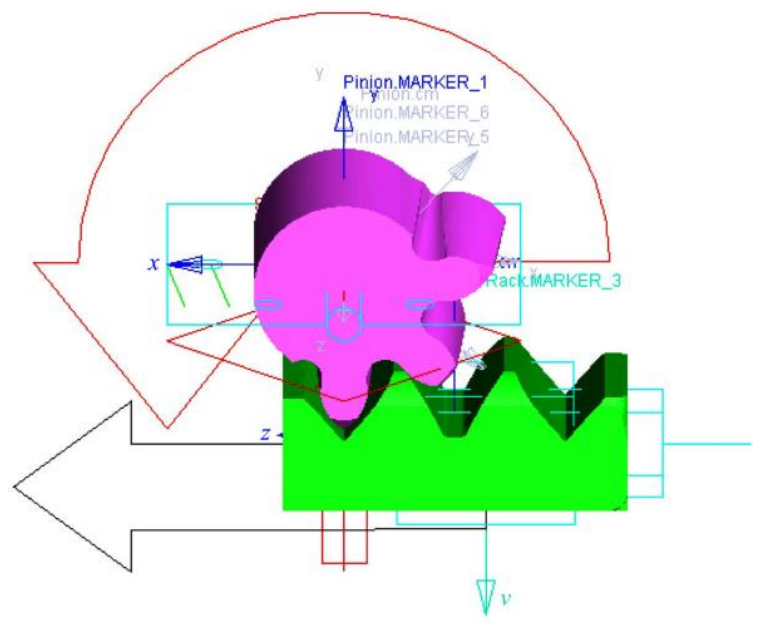

Figure 8 Simulation model of transmission stability

Four cases the initial VRTP obtained directly with the Boolean subtraction operation and the VRTPs modified by the B-spline curves of three different orders $(\lambda=3,4,5, \cdots)$ are discussed. The simulation results of the transmission ratio and transmission acceleration are shown in Figure 9 and Figure 10, respectively. The transmission ratio error (maximum error, total error and average error) and transmission acceleration error (maximum error and average error), which are calculated by theoretical values and simulate values, are collected in Table 1 and Table 2, respectively. 

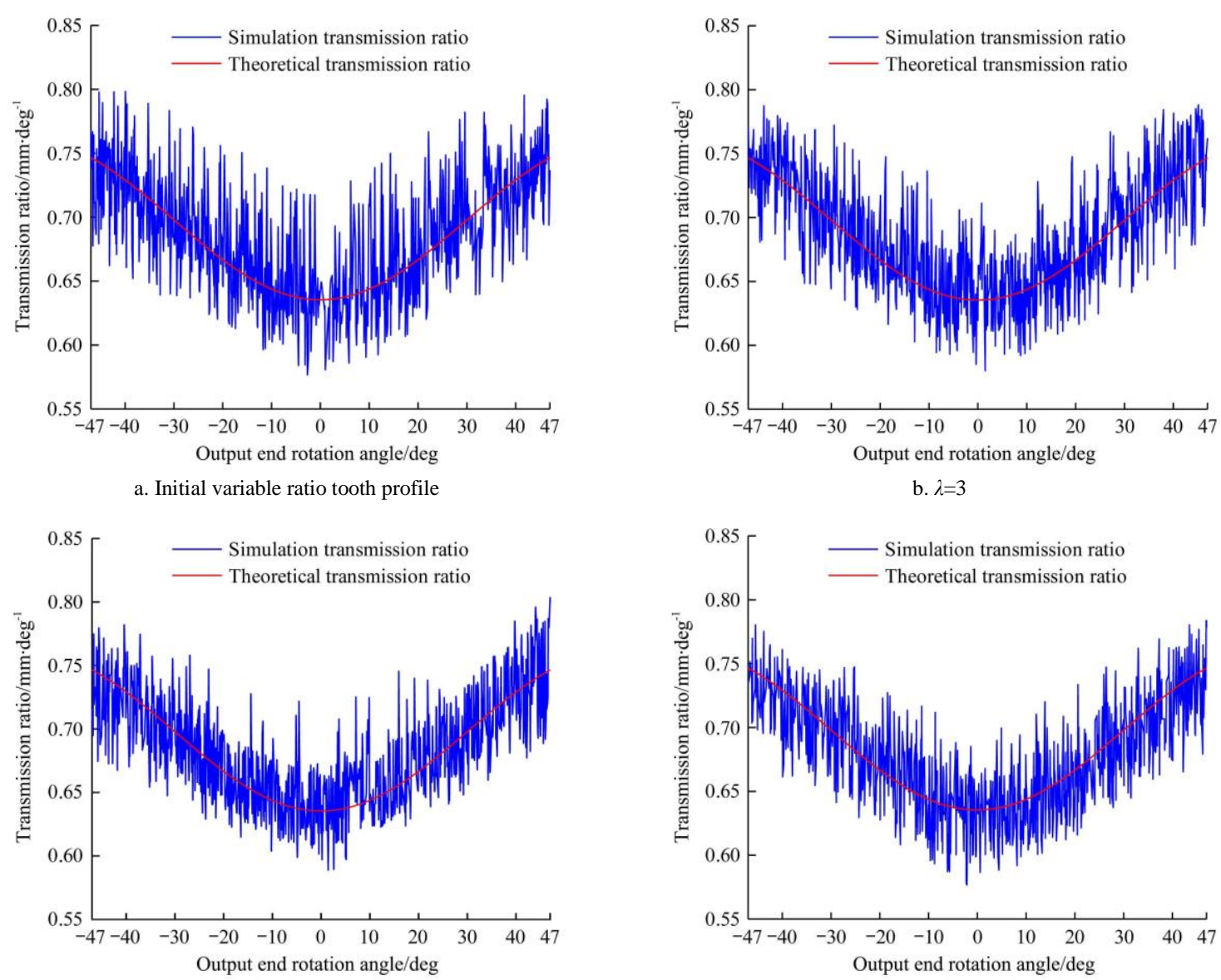

c. $\lambda=4$

d. $\lambda=5$

Figure 9 Simulation results of transmission ratio
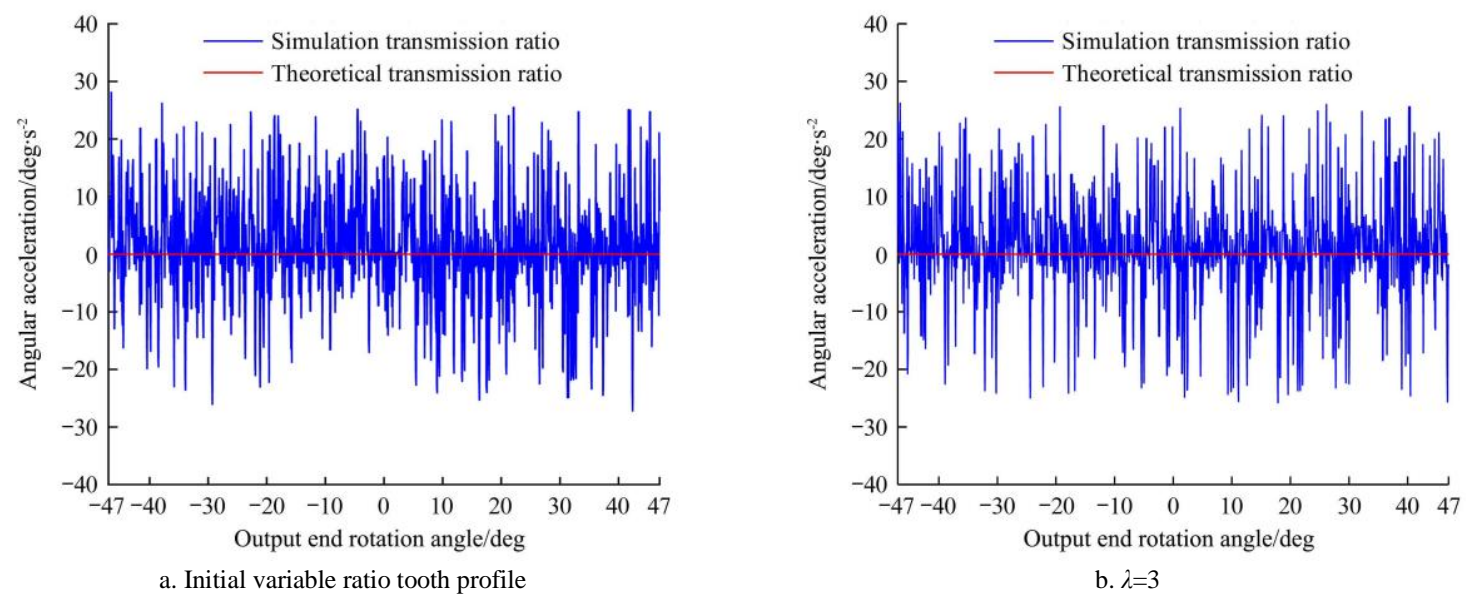

b. $\lambda=3$
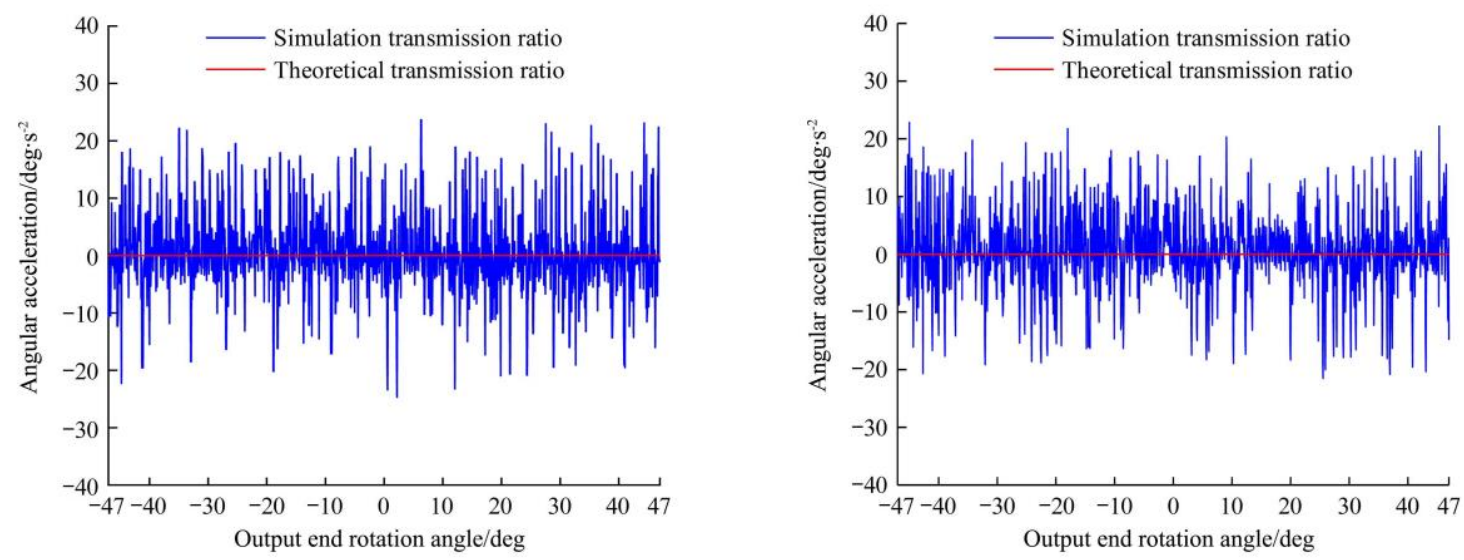

c. $\lambda=4$

d. $\lambda=5$

Figure 10 Simulation results of transmission acceleration 
Table 1 Transmission ratio error

\begin{tabular}{cccc}
\hline \multirow{2}{*}{ Objects } & \multicolumn{3}{c}{ Indexes } \\
\cline { 2 - 4 } & $\begin{array}{c}\text { Maximum } \\
\text { error/mm. }\left({ }^{\circ}\right)^{-1}\end{array}$ & $\begin{array}{c}\text { Maximum error } \\
\text { percentage/\% }\end{array}$ & $\begin{array}{c}\text { Average } \\
\text { error/mm } \cdot\left({ }^{\circ}\right)^{-1}\end{array}$ \\
\hline Initial & 0.096 & 12.38 & 0.025 \\
$\lambda=3$ & 0.092 & 12.47 & 0.023 \\
$\lambda=4$ & 0.087 & 11.73 & 0.023 \\
$\lambda=5$ & 0.068 & 9.54 & 0.022 \\
\hline
\end{tabular}

Table 2 Transmission acceleration error

\begin{tabular}{ccc}
\hline \multirow{2}{*}{ Objects } & \multicolumn{2}{c}{ Indexes } \\
\cline { 2 - 3 } & Maximum error $/\left({ }^{\circ}\right) \cdot \mathrm{s}^{-2}$ & Average error $/\left({ }^{\circ}\right) \cdot \mathrm{s}^{-2}$ \\
\hline Initial & 28.117 & 6.606 \\
$\lambda=3$ & 26.176 & 6.278 \\
$\lambda=4$ & 24.686 & 4.977 \\
$\lambda=5$ & 22.807 & 4.894 \\
\hline
\end{tabular}

As shown in Figure 9 and Figure 10, the simulation curves of the transmission ratio and the transmission acceleration of all modified VRTPs are generally a better approximation of the theoretical curves than those of the initial VRTP. For three different orders, the transmission stability simulation curves of the modified VRTP generated by the fifth order B-spline fitting curve have the smallest fluctuation level. In terms of quantitative analysis, according to the transmission ratio error (maximum error, total error and average error) shown in Table 1 and the transmission acceleration error (maximum error and average error) shown in Table 2, all indexes of the modified VRTPs are smaller than those of the initial VRTP. Meanwhile, these indexes confirm that transmission stability performs better as the order increases. However, as the order continues to increase $(\lambda>5)$, the simulation found that transmission stability will not present a distinct improvement, compared to the results of the fifth order. Therefore, the modified VRTP fitted by the fifth order B-spline curve with optimal transmission stability is selected as the final VRTP based on the above analyses. The results also illustrate the significant advantage of the modification method in overcoming the defects of Boolean subtraction modeling.

\section{Machining and testing}

\subsection{CNC machining of variable ratio tooth profile}

To validate the proposed design method, both numerical simulation and experimental verification should be employed. The numerical analysis and discussion in Section 3.3.2 play the role of numerical simulation. For now, based on computer-aided manufacturing (CAM) system, the final VRTP determined in Section 3.3.2 and the initial VRTP are manufactured virtually by end milling on universal three-axis milling machines, including rough machining, semi-finish and finish machining, as shown in Figure 11. The machining parameters are shown in Table 3.

After finishing the machining simulation and interference checking, the machining code for actual machining is entered into the three-axis CNC machine tool. Then, the machining sample of the rack with the final VRTP and the corresponding involute beveloid pinion-sector are shown in Figure 12.

\subsection{Testing and analysis of transmission stability}

Two testing gear pairs are constructed: one comprises the rack with the initial VRTP and the pinion-sector, while the other comprises the rack with the final VRTP and the pinion-sector. They are applied separately to the same steering to compare transmission stability. Per the introduction to Section 2, the tested steering consists of two-stage transmission pairs, and the transmission ratio of the screw-nut pair is $i_{s}=24\left(^{\circ}\right) / \mathrm{mm}$. Thus, the transmission ratio of the variable ratio rack and the pinion-sector can be recalculated as

$$
i=\frac{i_{z}}{i_{s}}=\left(\frac{d \beta}{d t}\right) /\left(C \cdot \frac{d \varphi}{d t}\right)
$$

where, $i_{z}$ is the total transmission ratio of the variable ratio steering; $\beta$ is the rotation angle of the input end, $\left(^{\circ}\right) ; \varphi$ is the rotation angle of the output end, $\left({ }^{\circ}\right)$.

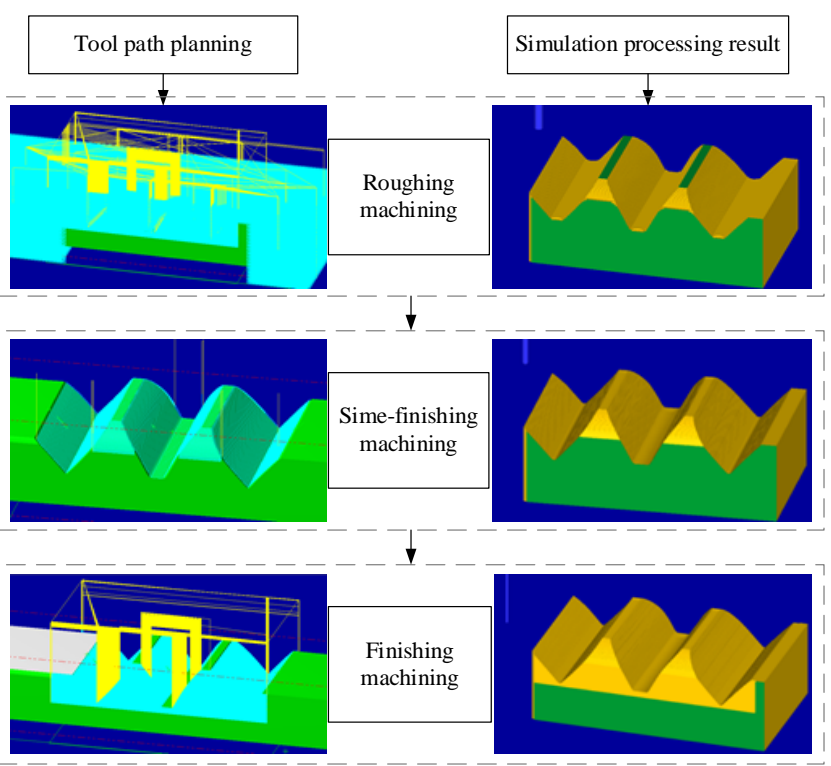

Figure 11 Simulation processing

Table 3 Variable ratio tooth profile processing parameters

\begin{tabular}{|c|c|c|c|}
\hline Item & Roughing & Semi-finishing & Finishing \\
\hline Machine type & 3-axis milling & 3-axis milling & 3-axis milling \\
\hline Milling style & Grooving roughing & Contour shape & Contour shape \\
\hline $\begin{array}{l}\text { Milling tool and diameter } \\
/ \mathrm{mm}\end{array}$ & Flat end, 6 & Ball end, 4 & Ball end, 3 \\
\hline $\begin{array}{l}\text { Maximum } Z \text {-axis feed } \\
/ \mathrm{mm}\end{array}$ & 1.50 & 1.00 & 0.75 \\
\hline Residual height $/ \mathrm{mm}$ & 4 & 1 & 0 \\
\hline Safe height $/ \mathrm{mm}$ & 20 & 20 & 20 \\
\hline Spindle speed/r $\cdot \min ^{-1}$ & 2000 & 2500 & 3000 \\
\hline Feed rate $/ \mathrm{mm} \cdot \mathrm{r}^{-1}$ & 0.8 & 0.6 & 0.2 \\
\hline
\end{tabular}

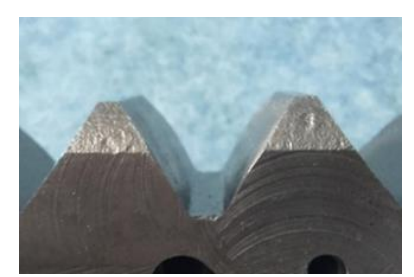

a. Rack with variable ratio tooth profile

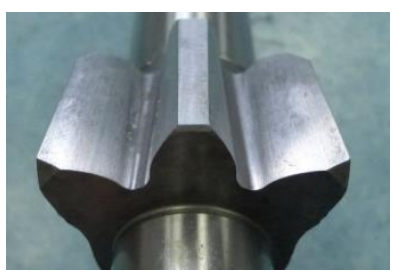

b. Pinion-secto
Figure 12 Samples of rack with variable ratio tooth profile and pinion-sector

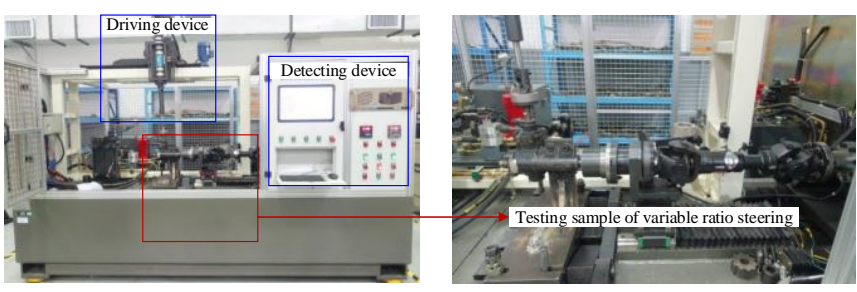

Figure 13 Test-bed of transmission stability 
The test-bed is shown in Figure 13, and mainly includes the driving device of the steering input end and the detecting device of the steering output end. The testing is carried out under the following conditions: rotation speed of the input end is $v_{s}=10 \mathrm{r} / \mathrm{min}$; a torque of $200 \mathrm{Nm}$ has been applied to the output end. Within a steering stroke, the instantaneous angle and the instantaneous

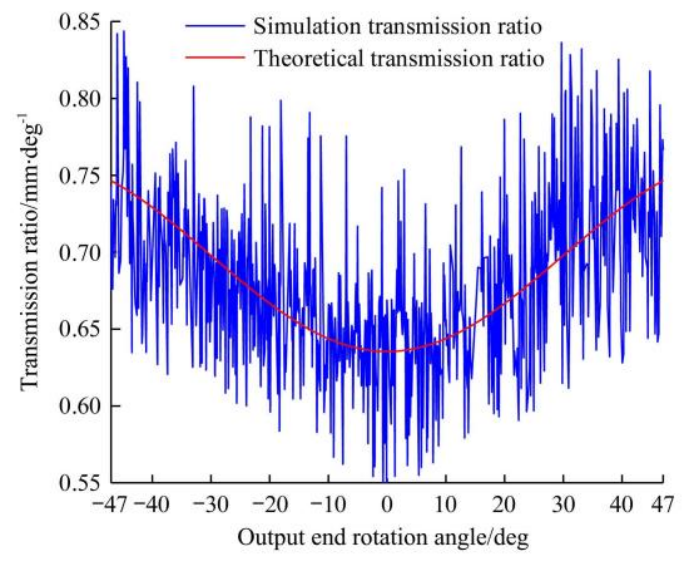

a. Initial variable ratio tooth profile acceleration of the output end are directly measured by an angle encoder and an angular acceleration sensor. Then the instantaneous angular velocity values of the output end are derived from the instantaneous angle values. Finally, the testing transmission ratio of the variable ratio rack and the pinion-sector pair can be obtained according to Equation (19).

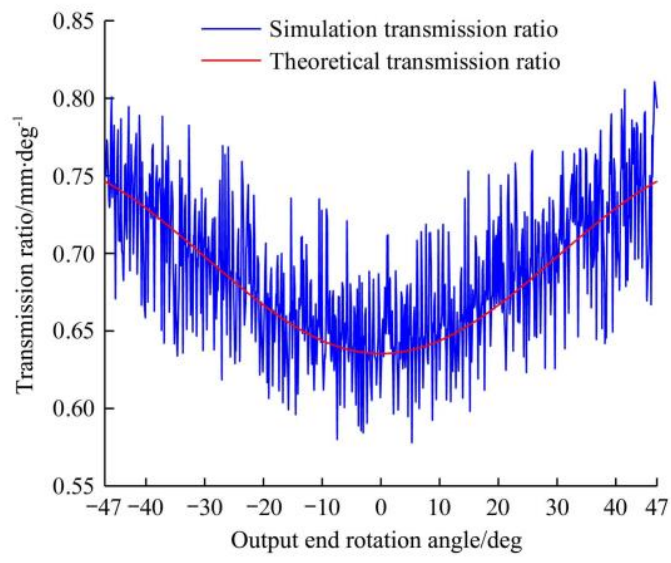

b. Final variable ratio tooth profile

Figure 14 Testing results of transmission ratio
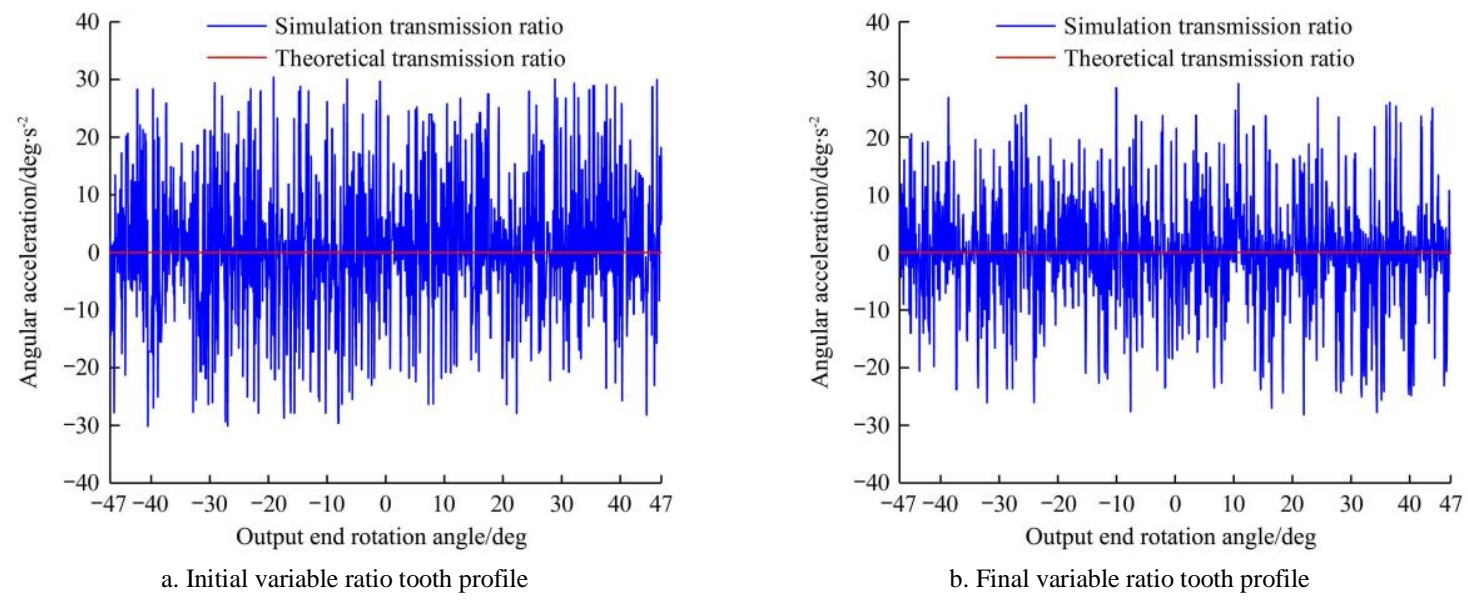

Figure 15 Testing results of transmission acceleration

The testing curves of the transmission ratio for the initial VRTP and the final VRTP are shown in Figure 14, and the testing curves of the transmission acceleration are shown in Figure 15. The fluctuation level of these curves testifies that the transmission stability of the final VRTP is better than that of the initial VRTP. After calculating the theoretical values and the testing values for the initial VRTP, the results of the transmission ratio error show that the maximum error is $0.14 \mathrm{~mm} / \mathrm{deg}$, the average error is $0.03 \mathrm{~mm} / \mathrm{deg}$, and the maximum error percentage is $17.61 \%$. The results of the transmission acceleration error show that the maximum error is $30.41\left(^{\circ}\right) / \mathrm{s}^{2}$, and the average error is $8.76\left(^{\circ}\right) / \mathrm{s}^{2}$. For the final VRTP, the results of the transmission ratio error show that the maximum error is $0.09 \mathrm{~mm} / \mathrm{deg}$, the average error is $0.02 \mathrm{~mm} / \mathrm{deg}$, and the maximum error percentage is $12.66 \%$. The results of the transmission acceleration error show that the maximum error is $29.30\left({ }^{\circ}\right) / \mathrm{s}^{2}$, and the average error is $6.48 \mathrm{deg} / \mathrm{s}^{2}$. The above results indicate that the modification method for improving the transmission stability of VRTP is feasible.

\section{Conclusions}

(1) Based on the Boolean subtraction operation, a solid modeling method for variable ratio tooth profiles is proposed. The initial steering rack with a discontinuous variable ratio tooth surface is generated directly, according to the discrete envelope motion relationship.

(2) A novel fitting algorithm is proposed to generate a continuous and smooth variable ratio tooth profile by using the B-spline curve to fit the approximate tooth profile points. Transmission stability simulations are conducted for the initial tooth profile obtained directly with the Boolean subtraction operation and the modified tooth profiles generated by B-spline curves of different orders. The results show that all of the modified tooth profiles are better than the initial tooth profile in transmission stability, and the variable ratio tooth profile with optimal transmission stability is selected as the final design.

(3) The transmission ratio and the transmission acceleration for the machining samples of the initial tooth profile and the final tooth profile are obtained by testing. The results indicate that the sample of the final variable ratio tooth profile is more effective than the sample of the initial variable ratio tooth profile. Furthermore, the testing shows that the modification method proposed in this paper is feasible. 


\section{Acknowledgements}

This work was financially supported by the Shandong Provincial Key Research and Development Program (Grant No. 2018GNC112017), Shandong Agricultural Machinery R\&D Innovation Project Sub-project (Grant No. 2018YF001-02), the Shandong Provincial Key Laboratory of Horticultural Machinery and Equipment (Grant No. YYJX-2019-08), the Funds of Shandong "Double Tops" Program (Grant No. SYL2017XTTD14), the Fundamental Research Funds for the Central Universities (Grant No. 2662020GXPY016) and the Hubei Provincial Natural Science Foundation of China (Grant No. 2018CFB231).

\section{[References]}

[1] Alexandru P, Macaveiu D, Alexandru C. A gear with translational wheel for a variable transmission ratio and applications to steering box. Mechanism and Machine Theory, 2012; 52: 267-276.

[2] Alexandru P. The rack-pinion gears for steering gear box with variable transmission ratio. Proceedings of the 12th IFToMM World Congress, Besancon, 2007; pp.353-358.

[3] Lee J, Yi K, Lee D, Jang B, Kim M, Hwang S. Haptic control of steer-by-wire systems for tracking of target steering feedback torque. Proceedings of the Institution of Mechanical Engineers, Part D: Journal of Automobile Engineering, 2020; 234(5): 1389-1401.

[4] Huang W, Wong P K, Wong K I, Vong C M, Zhao J. Adaptive neural control of vehicle yaw stability with active front steering using an improved random projection neural network. Vehicle System Dynamics, 2020; 58: 1-19.

[5] Zhang N, Wang M. Dynamic modeling of hydraulic power steering system with variable ratio rack and pinion gear. JSME International Journal Series C-Mechanical System Machine Elements and Manufacturing, 2005; 48(2): 251-260.

[6] Alexandru P, Ceausescu B, Diaconescu D. Important aspects of geometry of the pinion-rack gear with variable ratio. Proceedings of the 8th IFToMM International Symposium on Science of Mechanisms and Machines-SYROM, Bucharest, 2001; pp.13-18.

[7] Mao J H, Li L C, Wu X T, Zhang Z. The Numerical computation method of variable ratio rack conjugate surface. Chinese Journal of Mechanical Engineering, 2002; 5(5): 52-55. (in Chinese)

[8] Hu J, Fan W, Niu Z R, Li G Y. Digital design method of variable ratio gear for automobile recirculating ball variable ratio steering gear based on meshing theory. Journal of the Balkan Tribological Association, 2016; 22(1): 171-183.

[9] Hu J, Dong K K, Niu Z R, Li G Y. Digital design method of variable ratio gear pair for automobile mechanical variable ratio steering gear based on enveloping simulation. Journal of the Balkan Tribological Association, 2016; 22(1): 184-199.

[10] Xu M, Han X H, Hua L, Zheng F Y. Modeling and methods for gear shaping process and cutting force prediction of variable transmission ratio rack. International Journal of Mechanical Sciences, 2020; 171: 1389-1401.

[11] Zschippang H A, Weikert S, Küçük K A, Wegener K. Face-gear drive: Geometry generation and tooth contact analysis. Mechanism and Machine Theory, 2019; 142: $103576 . \quad$ doi: 10.1016/j.mechmachtheory.2019.103576.

[12] Feng G S, Xie Z F, Zhou M. Geometric design and analysis of face-gear drive with involute helical pinion. Mechanism and Machine Theory, 2019; 134: 169-196.

[13] Guo H, Gonzalez-Perez I, Fuentes-Aznar A. Computerized generation and meshing simulation of face gear drives manufactured by circular cutters. Mechanism and Machine Theory, 2019; 133: 44-63.

[14] Xiang T M, Gu L Z, Xiao L L. Accurate modeling of logarithmic spiral bevel gear based on the tooth flank formation and Boolean addition operation. Proceedings of the Institution of Mechanical Engineers, Part B : Journal of Engineering Manufacture, 2016; 230(9): 1650-1658.

[15] Liu S Y, Song C S, Zhu C C, Ni G X. Effect of tooth modifications on mesh characteristics of crossed beveloid gear pair with small shaft angle. Mechanism and Machine Theory, 2018; 119: 142-160.

[16] Fu X Z, Fang Z D, Cui Y M, Hou X Y, Li J H. Modelling, design and analysis of offset, non-orthogonal and profile-shifted face gear drives. Advances in Mechanical Engineering, 2018; 10(9): 1-12.

[17] Zheng F Y, Han X H, Hua L, Zhang M D, Zhang W Q. Design and manufacture of new type of non-circular cylindrical gear generated by face-milling method. Mechanism and Machine Theory, 2018; 122: 326-346.

[18] Luo S M, Liao L X, Wang J, Wang Y, Yi J X. Study on inspection and avoidance of interferences in five-axis end milling of cycloidal gears. The International Journal of Advanced Manufacturing Technology, 2017; 91(9-12): 3307-3314.

[19] Litvin F L, Feng P H. Computerized design, generation, and simulation of meshing of rotors of screw compressor. Mechanism and Machine Theory, 1997; 32(2): 137-160.

[20] Litvin F L, Fuentes A, Gonzalez-perez L, Carvenali L, Kawasaki K, Handschuh R F. Modified involute helical gears: computerized design, simulation of meshing and stress analysis. Comput. Methods Appl. Mech. Engrg., 2003; 192(33-34): 3619-3665.

[21] Litvin F L, Gonzalez-Perez I, Fuentes A, Hayasaka K, Yukishima K. Topology of modified surfaces of involute helical gears with line contact developed for improvement of bearing contact, reduction of transmission errors, and stress analysis. Mathematical and Computer Modelling, 2005; 42(9-10): 1063-1078.

[22] Litvin F L, Gonzalez-Perez I, Yukishima K, Fuentes A, Hayasaka K. Generation of planar and helical elliptical gears by application of rack-cutter, hob, and shaper. Computer Methods in Applied Mechanics and Engineering, 2007; 196(41): 4321-4336.

[23] Litvin F L, Gonzalez-Perez I, Fuentes A, Hayasaka K. Design and investigation of gear drives with non-circular gears applied for speed variation and generation of functions. Comput. Methods Appl. Mech. Engrg., 2008; 197: 3783-3802.

[24] Liu D W, Ren T Z, Jin X. Geometrical model and tooth analysis of undulating face gear. Mechanism and Machine Theory, 2015; 86: 140-155.

[25] Wu Y H, Zhou Y S, Zhou Z Y, Tang J Y, Ouyang H W. An advanced $\mathrm{CAD} / \mathrm{CAE}$ integration method for the generative design of face gears. Advances in Engineering Software, 2018; 126: 90-99.

[26] Zhou Y S, Wang S H, Wang L M, Tang J Y, Chen Z Z. CNC milling of face gears with a novel geometric analysis. Mechanism and Machine Theory, 2019; 139: 46-65.

[27] Mazak J, Klocke F, Bergs T, Brecher C, Löpenhaus C. Simulation-based process analysis for discontinuous cutting of generated bevel gears. Proceedings of the Institution of Mechanical Engineers, 2019; 233(21-22): 7378-7390.

[28] Guo E K, Hong R J, Huang X D, Fang C G. Research on the design of skiving tool for machining involute gears. Journal of Mechanical Science and Technology, 2014; 28(12): 5107-5115.

[29] Lin C, Xia X G, Li P L. Geometric design and kinematics analysis of coplanar double internal meshing non-circular planetary gear train. Advances in Mechanical Engineering, 2018; 10(12): 1-12.

[30] De Boor C. A Practical Guide to Splines. New York: Springer-Verlag, $1978 ; 346$. 